\title{
High-density SNP mapping reveals closely linked QTL for resistance to Stagonospora nodorum blotch (SNB) in flag leaf and glume of hexaploid wheat.
}

\begin{tabular}{|c|c|}
\hline Journal: & Genome \\
\hline Manuscript ID & gen-2017-0203.R1 \\
\hline Manuscript Type: & Note \\
\hline Date Submitted by the Author: & 28-Nov-2017 \\
\hline Complete List of Authors: & $\begin{array}{l}\text { Francki, Michael; Department of Agriculture and Food Western Australia, ; } \\
\text { Murdoch University, State Agricultural Biotechnology Centre } \\
\text { Walker, Esther ; Western Australia Department of Agriculture and Food, } \\
\text { Grains R\&D Transformation } \\
\text { Li, Dora ; DAFWA, Biotechnology } \\
\text { Forrest, Kerrie; Agriculture Victoria Research, Department of Economic } \\
\text { Development, Jobs, Transport and Resources }\end{array}$ \\
\hline $\begin{array}{r}\text { Is the invited manuscript for } \\
\text { consideration in a Special } \\
\text { Issue? : }\end{array}$ & $\mathrm{N} / \mathrm{A}$ \\
\hline Keyword: & $\begin{array}{l}\text { Stagonospora nodorum blotch, quantitative trait loci, adult plant } \\
\text { resistance, single nucleotide polymorphism }\end{array}$ \\
\hline
\end{tabular}

\section{SCHOLARONE ${ }^{m}$ \\ Manuscripts}


1 High-density SNP mapping reveals closely linked QTL for resistance to

2 Stagonospora nodorum blotch (SNB) in flag leaf and glume of hexaploid

3 wheat.

4

5 Michael G. Francki • Esther Walker • Dora A. Li • Kerrie Forrest

6

7

Michael G. Francki $\bowtie \cdot$ Esther Walker

8

Department of Agriculture and Food Western Australia, South Perth WA 6151, Australia

9

Michael G. Francki $\bowtie \cdot$ Esther Walker • Dora A. Li

Kerrie Forrest 


\section{Abstract}

The genetic control of adult plant resistance to Stagonospora nodorum blotch (SNB) is complex consisting of genes with minor effects interacting in an additive manner. Earlier studies detected quantitative trait loci (QTL) for flag leaf resistance in successive years on chromosomes 1B, 2A, 2D, 5B using SSR- and DArT-based genetic maps of progeny from the crosses EGA Blanco/Millewa, 6HRWSN125/WAWHT2074 and P92201D5/P91193D1. Similarly, QTL for glume resistance detected in successive years and multiple environments were identified on chromosomes 2D and 4B from genetic maps of P92201D5/P91193D1 and 6HRWSN125/WAWHT2074, respectively. The SSR- and DArT-based genetic maps had an average distance of $6.5,7.8$ and $9.7 \mathrm{cM}$ between marker loci for populations EGA/Millewa, P92201D5/P91193D1 and 6HRWSN125/WAWHT2074, respectively. This study used single nucleotide polymorphism (SNP) markers from the iSelect Infinium 90K genotyping array to fine map genomic regions harbouring QTL for flag leaf and glume SNB resistance reducing the average distance between markers to $2.9,3.3$ and $3.4-3.4 \mathrm{cM}$ for populations P92201D5/P91193D1, EGA/Millewa and 6HRWSN125/WAWHT2074, respectively. Increasing the marker density of the genetic maps with SNPs did not identify any new QTL for SNB resistance but discriminated previously identified co-located QTL into separate but closely linked QTL.

Key words: Stagonospora nodorum blotch; quantitative trait loci; adult plant resistance; single nucleotide polymorphism 


\section{Introduction}

Stagonospora nodorum blotch (SNB) is a nercrotophic fungal disease of wheat caused by the pathogen Parastagonospora nodorum (synonyms Stagonospora nodorum, Phaeosphaeria nodorum). Symptoms of infection in adult plants generally develop in wet and humid environments and include red-brown lesions surrounded by a yellow halo on flag leaves or pale to brown lesions on the glume. SNB infection on adult plants reduces the photosynthetic capacity of flag leaves, heads and peduncle causing significant losses in grain yield (Krupinsky et al 1973). Although fungicide application and crop rotation are common approaches for managing SNB, the use of resistant wheat varieties provides a complementary strategy to maintain grain yields under SNB epidemics.

Breeding for SNB resistance in wheat is a challenging task due to complex quantitative genetic control. Genetic analysis for adult plant resistance in spring and winter wheat has identified several minor quantitative trait loci (QTL) interacting in an additive manner, where genes expressing resistance in flag leaves are independent to those in glumes (reviewed in Francki 2013). QTL for flag leaf resistance on chromosomes $1 \mathrm{~B}, 2 \mathrm{~A}, 2 \mathrm{D}$ and $5 \mathrm{~B}$ were detected in successive years under Australian field conditions using spring wheat doubled-haploid populations EGA Blanco/Millewa (Francki et al. 2011) and 6HRWSN125/WAWHT2074 (Shankar et al. 2008), while QTL for glume resistance were detected on chromosome 2D in the latter population (Shankar et al. 2008). Analysis of glume response in a P92201D5/P91193D1 recombinant-inbred population across multiple environments detected the same QTL on chromosome 2D (Uphaus et al. 2007), suggesting similar genes may be responsible for expression of resistance to the pathogen in USA and Australia. It was therefore reasonable to assume that co-located QTL for flag leaf and glume resistance in the EGA Blanco/Millewa, 6HRWSN125/WAWHT2074 and P92201D5/P91193D1 populations contained the same genes responding to SNB infection in different environments. However, the genetic maps underpinning those studies were based on a maximum of 492 DNA markers, the average distance between marker loci was as high as 9.7 cM (Francki et al. 2009; Shankar et al. 2008), and QTL intervals spanned $10-30 \mathrm{cM}$ and accounted for 8-38\% of the phenotypic variation (reviewed in Francki 2013). 
QTL spanning large genome intervals (i.e. $>5 \mathrm{cM}$ ) can have restrictions when transferred to breeders germplasm. In particular is the increased risk of chromosome recombination between marker loci and the gene when QTL intervals are large which reduces the accuracy of selection for desirable phenotype. To mitigate these problems, fine mapping can be used to reduce QTL intervals and thereby increase efficiencies for QTL introgression in breeding programs (Salvi and Tuberosa 2005). Recently, several high-density genotyping arrays providing wide genome coverage have been developed for wheat including the iSelect Infinium 9K and 90K bead chip arrays (Cavanagh et al. 2013; Wang et al. 2014). Here, we used the iSelect 90K wheat SNP array to genotype the EGA Blanco/Millewa, 6HRWSN125/WAWHT2074 and the P92201D5/P91193D1 populations to create a high-density integrated SNP, SSR and DArT genetic map for each population to help refine QTL intervals for flag leaf and glume resistance to SNB.

\section{Methods}

SNP genotyping and genetic map construction

DNA samples from 241, 250 and 239 individuals of doubled-haploid (DH) populations EGA Blanco/Millewa and 6HRWSN125/WAWHT2074 and recombinant-inbred (RI) line population P92201D5/P91193D1, respectively, were assayed using the 90K iSelect SNP array Agriculture Victoria Research, Agribio, Bundoora, VIC, Australia. SNP clustering and assignment of samples to cluster was performed using custom scripts. In brief, sample theta and normR values for each SNP were exported from GenomeStudio v1.9.4. Assignment of a sample to a cluster required that the sample data point fell within two standard deviations of the sample cluster, and that the confidence score for assignment to that cluster versus the next closest cluster exceeded the value of 0.7 , where 0 and 1 represent lowest and highest confidence, respectively. Cluster assignments were converted to binary parental phasing using a custom perl script. Genotyping data for each population was preliminarily mapped using MSTmap (Wu et al. 2008) at LOD6 to assess the quality of genotype calling. Markers with either less than $80 \%$ call rate or segregation distortion ( $p$-value $>0.10$ calculated from a chi-square test) were removed. Moreover, samples with a high crossover rate (indicating a 
mixed sample) or duplicate samples (>95\% identical genotypes) were also removed. The datasets for each population were then merged with SSR, DArT and other markers previously genotyped for these populations (Francki et al. 2011; Shankar et al. 2008; Uphaus et al. 2007) and re-mapped using MSTmap at LOD6, without redundant markers, selecting "DH" as Population Type for populations EGA Blanco/Millewa and 6HRWSN125/WAWHT2074, and "RIL at generation 8" for population P92201D5/P91193D1. The merged datasets were also mapped using QTL Ici Mapping Version 4.0.6.0 (Meng et al. 2015) to confirm marker order. Redundant markers were removed by selecting the BIN parameter within QTL IciMapping using "Delete redundancy By Missing Rate (\%)" and missing data was set at $20 \%$ using the "Delete markers By missing rate (\%)" function. MAP parameters were set at LOD6, specifying Algorithm: nnTwoOpt and Rippling:SARF.

QTL mapping with SNP-based genetic maps

The high-density genetic maps containing SNP markers and mean flag leaf and glume disease scores from multi-environments (Francki et al. 2011; Shankar et al. 2008; Uphaus et al. 2007) were used to identify any new QTL not previously detected using SSR and DArT-based genetic maps and to fine map existing genomic regions for SNB resistance. QTL IciMapping was used to identify QTL regions using inclusive composite interval mapping with additive effect (ICIM-ADD). Parameters for all traits and environments were set to $0.1 \mathrm{cM}$ steps, Probability in Stepwise Regression (PIN) of 0.001 , minimum LOD threshold of 2.5 and deleting missing phenotypes. Final maps and associated QTL were drawn using MapChart Version 2.3 (Voorrips, 2002).

\section{Results and Discussion}

The $90 \mathrm{~K}$ iSelect SNP array substantially increased the number of markers with identification of 8,760-16,411 polymorphic SNP loci (Table 1) representing 22.8-33.3 times the number of existing markers for each genetic map reported in Francki et al. (2009) and Shankar et al. (2008). However, the number of non-redundant SNP markers polymorphic between parents of the mapping populations ranged from 1,183-1,767 (Table 1) representing 8-20\% of the total polymorphic SNP markers and were used to integrate with other markers in genetic maps. Although the SNP markers cover the 
majority of the wheat genome, the high SNP redundancy reflects the marker density to the limited recombination for each $\mathrm{DH}$ and RIL population. The total number of SSR, DArT, non-redundant SNP and other markers mapped in the EGA Blanco/Millewa, 6HRWSN125/WAWHT2074 and P92201D5/P91193D1 populations were 1,459, 1,713 and 2,047, respectively, where the integration of non-redundant polymorphic SNP markers expanded the total genetic map distance 4,906-6,022 cM (Table 1) from the SSR- and DArT-based map reported in Francki et al. 2009 and Shankar et al. 2008. The average genetic map distance between SNP and other marker loci was $2.94 \mathrm{cM}, 3.36 \mathrm{cM}$ and 3.43 cM for populations P92201D5/P91193D1, EGA/Millewa and 6HRWSN125/WAWHT2074, respectively (Table 1). The intervals were reduced from 6.5, 7.8 and 9.7 cM between marker loci for populations EGA/Millewa, P92201D5/P91193D1 and 6HRWSN125/WAWHT2074, respectively, when predominantly SSR and DArT markers were used in map construction (Francki et al. 2009; Shankar et al. 2008). SNP marker locus intervals were comparable to previously published high-density SNP. based marker maps derived from the $\mathrm{DH}$ mapping populations of hexaploid wheat, Avalon/Cadenza and Savannah/Rialto, with average intervals of 2.8 and $3.8 \mathrm{cM}$, respectively (Allen et al. 2011; Allen et al. 2013). The smaller interval size was, therefore, suitable for fine mapping QTL for SNB resistance in flag leaf and glumes.

The co-location of QTL using SSR and DArT marker-based genetic maps indicated that genome regions controlling SNB resistance contain similar genes expressed in different environments. QTL analysis of maps supplemented with SNP markers did not identify any new QTL for flag leaf resistance. It was anticipated that existing QTL for flag leaf resistance detected in different environments would co-locate in the same genomic region as previously described in Francki et al. (2011) and Shankar et al. (2008) using genetic maps supplemented with SNP markers. Instead, the higher density genetic maps discriminated two closely linked QTL for flag leaf resistance detected in different environments on chromosome 2A and 2D from the populations P92201D5/P91193D1 and 6HRWSN125/WAWHT2074, respectively (Fig. 1). The distinct QTL provided unambiguous evidence that multiple flag leaf genes on chromosomes $2 \mathrm{~A}$ and $2 \mathrm{D}$ are linked within $17 \mathrm{cM}$ and $5 \mathrm{cM}$, respectively, and express resistance in response to different environmental signals. The proportion of 
phenotypic variation accounted by each QTL using genetic maps supplemented with SNP markers ranged from $7.8 \%$ to $20.8 \%$ depending on the environment (Fig. 1). The proportion of variation accounted for by each QTL and environment using composite interval mapping was the same as previously reported in Francki et al. (2011) and Shankar et al. (2008).

Similarly, SNP markers were able to discriminate at least two QTL for flag leaf resistance on chromosomes 5B in the EGA Blanco/Millewa population where each QTL contained genes that express resistance in response to different environments (Fig. 1). However, additional SNP markers within a 10-cM interval were unable to discriminate between the QsnI08.daw QTL for flag leaf SNB resistance detected in 2008 and Qhd07.daw and Qhd08.daw for heading date in 2007 and 2008 on chromosome 5B (Fig. 1). As heading date was unlikely to have pleiotropic effects on flag leaf resistance in the EGA Blanco/Millewa population (Francki et al. 2011) it is anticipated, therefore, that further fine mapping within this region would discriminate QTL for flag leaf resistance from heading date. Although high-density SNP markers were mapped on chromosome 1B in the EGA Blanco/Millewa population (Fig. 1), it remains unclear whether single or linked QTL controlling flag leaf resistance in different environments reside at this locus. Analysis using a larger size of the EGA Blanco/Millewa population would determine whether single or multiple QTL for flag leaf resistance reside on chromosome $1 \mathrm{~B}$.

The genetic maps supplemented with SNP markers were used in QTL analysis for SNB resistance in glumes for the 6HRWSN125/WAWHT2074 population using mean glume disease scores from two environments reported in Shankar et al (2008). Glume resistance on chromosome 4B identified two linked QTL within $12 \mathrm{cM}$ (Fig. 2), indicating at least two genes underpin resistance and are expressed in this genomic region. The QTL explained similar phenotypic variance to that reported previously by Shankar et al. (2008). However, high-resolution SNP mapping detected QTL co-located for glume resistance on chromosome 2D within the same SNP marker interval of $0.3 \mathrm{cM}$ (IWB31450- IWB21124) when the P92201D5/P91193D1 population was evaluated in three environments in the USA (Fig. 2). Distinct but tightly linked QTL were also detected for glume resistance in two environments in Australia and resided in the (Xgwm526a - IWB53436) marker 
interval of $<1.0 \mathrm{cM}$ (Fig. 2). Therefore, it appears that the same genes control glume resistance to SNB across different USA environments but are different to resistance genes that consistently respond to variable Australian environments.

The significant increase in map density provided by SNP markers in three genetic maps in this study provided a new insight on the QTL underpinning SNB resistance contributing to the genetic complexity of this trait in wheat. It was reasonable to assume in earlier studies (Francki et al. 2011; Shankar et al. 2008; Uphaus et al 2007) that similar genes controlled expression of flag leaf and glume resistance to SNB in different environments based on the co-location of QTL using mediumresolution SSR- and DArT-based genetic maps. Instead, fine mapping using SNP markers in this study revealed multiple but closely linked QTL, indicating distinct clusters of concomitant diseaserelated genes in the host that respond differently to pathogen infection across environments. Plant resistance $(R)$ multi-gene families are typically tandem or segmentally duplicated for large stretches of contiguous DNA and contain diverse copies within clusters that have evolved through selective forces generating novel or diverse pathogen recognition capabilities (Gururani et al. 2012; Leister 2004; Liu et al. 2007; Meyers et al. 2005). Therefore, it is conceivable that multiple linked QTL for SNB resistance identified in this study through high-density SNP mapping may have detected disease response from clusters of $R$ genes or $R$ gene-mediated signalling pathway factors that respond to specific isolates in mixed inoculum that predominate in specific environments. The identified SNP markers in this study would assist in aligning QTL intervals to the sequenced genome of wheat (The International Wheat Genome Sequencing Consortium, 2014) and provide further knowledge of the underlying genes involved in SNB resistance. In the meantime, the knowledge of the genetic control and SNP markers identified in this study are exemplary tools to introgress clusters of disease related genes for breeding improved SNB resistance in wheat.

Acknowledgements: Funding from the Grains Research Development Corporation through DAW00248 and DAV00127 is gratefully acknowledged.

\section{References}


Allen, A., Barker, G., Berry, S., Coghill, J., Gwilliam, R., Kirby, S., Robinson, P. et al. 2011. Transcriptspecific, single-nucleotide polymorphism discovery and linkage analysis in hexaploid bread wheat (Tricticum aestivum L.). Plant Biotech. J. 9:1086-1099

Allen, A., Barker, G., Wilkinson, P., Burridge, A., Winfield, M., Coghill, J., Uauy, C. et al. 2013. Discovery and development of exome based, co-dominant single nucleotide polymorphism markers in hexaploid wheat (Tricticum aestivum L.). Plant Biotech. J. 11:279-295

Cavanagh, C., Chao, S., Wang, S., Huang, B.E., Stephen, S., Kiani, S., Forrest, K. et al. 2013. Genome-wide comparative diversity uncovers multiple targets of selection for improvement in hexaploid wheat landraces and cultivars. Proc. Natl. Acad. Sci. USA 110:8057-8062

Francki, M. 2013. Improving Stagonospora nodorum resistance in wheat: a review. Crop Sci .53:355365

Francki, M., Walker, E., Crawford, A., Broughton, S., Ohm, H., Barclay, I., Wilson, R., McLean, R. 2009. Comparison of genetic and cytogenetic maps of hexaploid wheat (Triticum aestivum L.) using SSR and DArT markers. Mol. Genet. Genomics 281:181-191

Francki, M., Shankar, M., Walker, E., Loughman, R., Golzar, H., Ohm, H. 2011. New quantitative trait loci for flag leaf resistance to Stagonospora nnodorum blotch. Phytopath. 101:1278-1284

Gururani, M., Venkatesh, J., Upadhyaya, C., Nookaraju, A., Pandey, S., Park, S. 2012. Plant disease resistance genes: current status and future directions. Physiol. Mol. Plant Pathol. 78:51-65

Krupinsky, J., Scharen, A., Schillinger, J. 1973. Pathogenic variation in Septoria nodorum (Berk.) Berk. in relation to organ specificity, apparent photosynthetic rate and yield of wheat. Physiol. Plant Path. 3:187-194

Leister, D. 2004. Tandem and segmental duplication and recombination in the evolution of plant disease resistance genes. Trends Genet. 20:116-122 
Liu, J., Liu, X., Dai, L., Wang, G. 2007. Recent progress in elucidating the structure, function and evolution of disease resistance genes in plants. J. Genet. Genom. 34:765-776

Meng, L., Li, H., Zhang, L., Wang, J. 2015. QTL IciMapping: Integrated software for genetic linkage map construction and quantitative trait locus mapping in biparental populations. Crop J. 3: 269283

Myers, B., Kaushik, S., Nandety, R. 2005. Evolving disease resistance genes. Curr. Op. Plant Biol. 8:129-134

Salvi, S., Tuberosa, R. 2005. To clone or not to clone plant QTLs: present and future challenges. Trends Plant Sci. 10:297-304

Shankar, M., Walker, E., Golzar, H., Loughman, R., Wilson, R., Francki, M. 2008. Quantitative trait loci for seedling and adult plant resistance to Stagonospora nodorum in wheat. Phytopath. $98: 886-893$

The International Wheat Genome Sequencing Consortium (IWGSC) 2014. A chromosome-based draft sequence of the hexaploid bread wheat (Triticum aestivum) genome. Science 345:1251788

Uphaus, J., Walker, E., Shankar, M., Golzar, H., Loughman, R., Francki, M., Ohm, H. 2007. Quantitative trait loci to Stagonospora glume blotch in wheat in the USA and Australia. Crop Sci. $47: 1813-1822$

Voorrips, R. 2002. MapChart: Software for the graphical presentation of linkage maps and QTLs. J Hered. 93:77-78

Wang, S., Wong, D., Forrest, K., Allen, A., Chao, S., Huang, B., Maccaferri, M. et al. 2014. Characterization of polyploid wheat genomic diversity using a high-density 90000 single nucleotide polymorphism array. Plant Biotech. J. 12:787-796

Wu, Y., Bhat, P., Close, T., Lonardi, S. 2008. Efficient and accurate construction of genetic linkage maps from the minimum spanning Tree of a Graph. PLoS Genet. 4: e1000212 
Table 1 Summary of total and non-redundant (NR) SNP and other DNA markers mapped in three homozygous bi-parental mapping populations used for QTL analysis for SNB resistance in hexaploid wheat.

\begin{tabular}{lccc}
\hline & EGA Blanco/Millewa & 6HRWSN125/WAWHT2074 & P92201D5/P91191D1 \\
\hline SNP markers & 13,308 & 16,411 & 8,760 \\
Other markers & $468^{1}$ & $492^{2}$ & $385^{1}$ \\
Total markers & 13,776 & 16,903 & 9,145 \\
NR SNP markers & 1,183 & 1,346 & 1,767 \\
NR other markers & 276 & 367 & 280 \\
Total NR markers & 1,459 & 1,713 & 2,047 \\
Total map distance & $4,906 \mathrm{cM}$ & $5,885 \mathrm{cM}$ & $6,022 \mathrm{cM}$ \\
Average map distance between markers & $3.36 \mathrm{cM}$ & $3.43 \mathrm{cM}$ & $2.94 \mathrm{cM}$ \\
\hline
\end{tabular}

${ }^{1}$ Other markers described in Francki et al (2009)

'Other markers described in Shankar et al (2008) 
Fig. 1 QTL analysis for flag leaf resistance to SNB from populations EGA Blanco/Millewa (chromosomes 1B and 5B), 6HRWSN125/WAWHT2074 (chromosome 2D) and P92201D5/P91191D1 (chromosome 2A). Chromosomal location of SNP integrated with other DNA markers are shown to the left and QTL traces based on phenotypic data for flag leaf response from successive years are shown on the right. Coloured solid bars in traces represent QTL regions detected in different environments ( $Q n /$ designations) and threshold LOD are represented by dotted lines. The phenotypic variance accounted for each QTL is shown in parentheses.

Fig. 2 QTL analysis for glume resistance to SNB from populations P92201D5/P91191D1 (chromosome 2D) and 6HRWSN125/WAWHT2074 (chromosome 4B). Chromosomal location of SNP integrated with other DNA markers are shown to the left and QTL traces based on phenotypic data for glume response from successive years screened in Australia and USA are shown on the right. Coloured solid bars in traces represent QTL regions detected in Australian and USA environments (Qng.daw and Qng.pur designations, respectively) and threshold LOD are represented by dotted lines. The phenotypic variance accounted for each QTL is shown in parentheses. 
Chromosome 1B
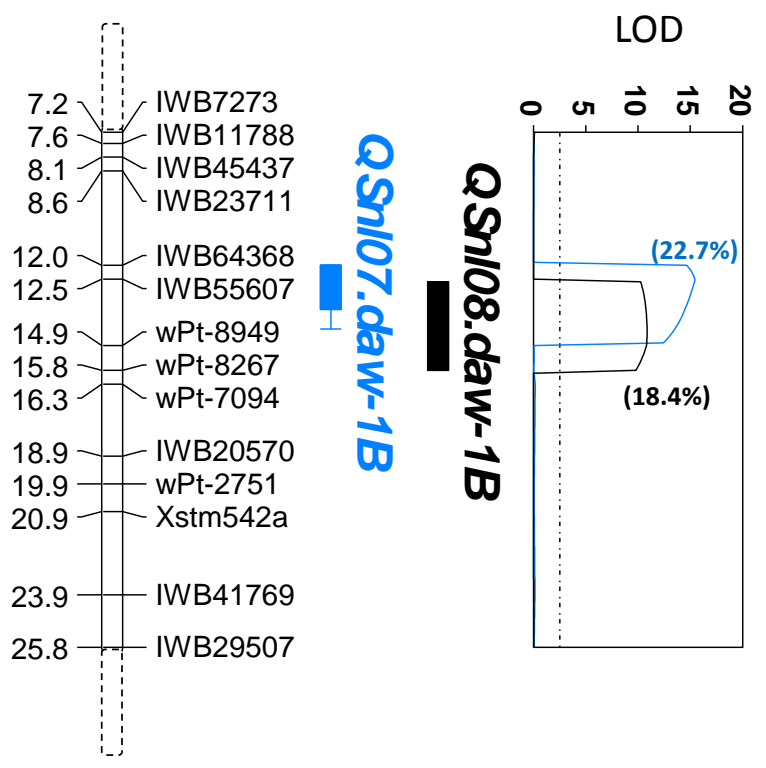

Chromosome 2A
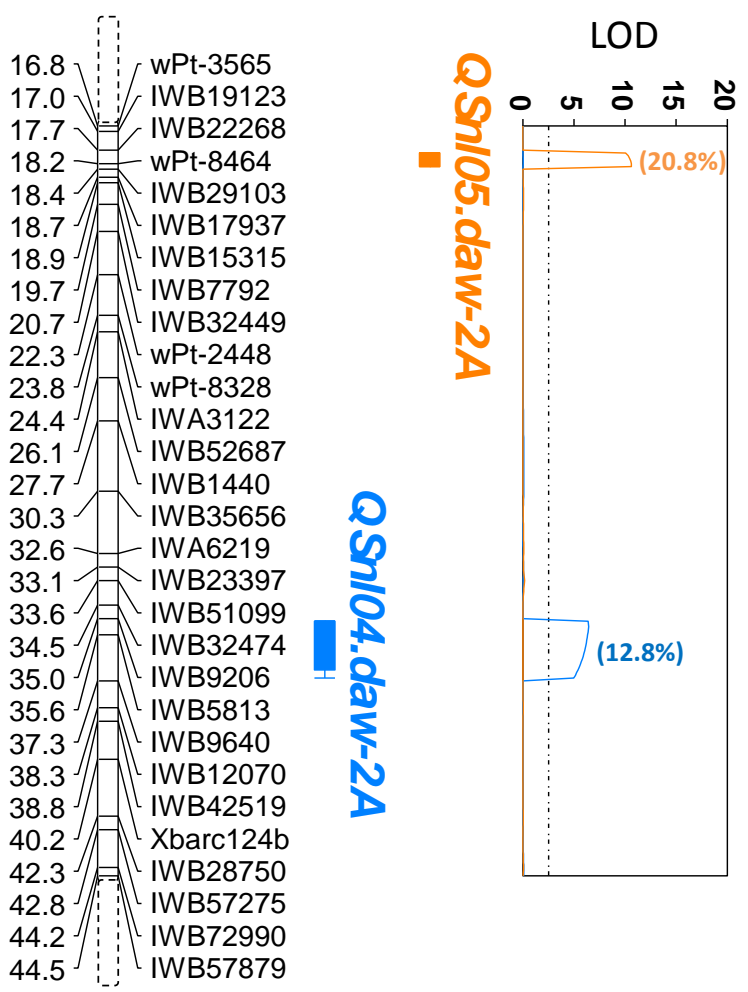

Chromosome 5B

LOD

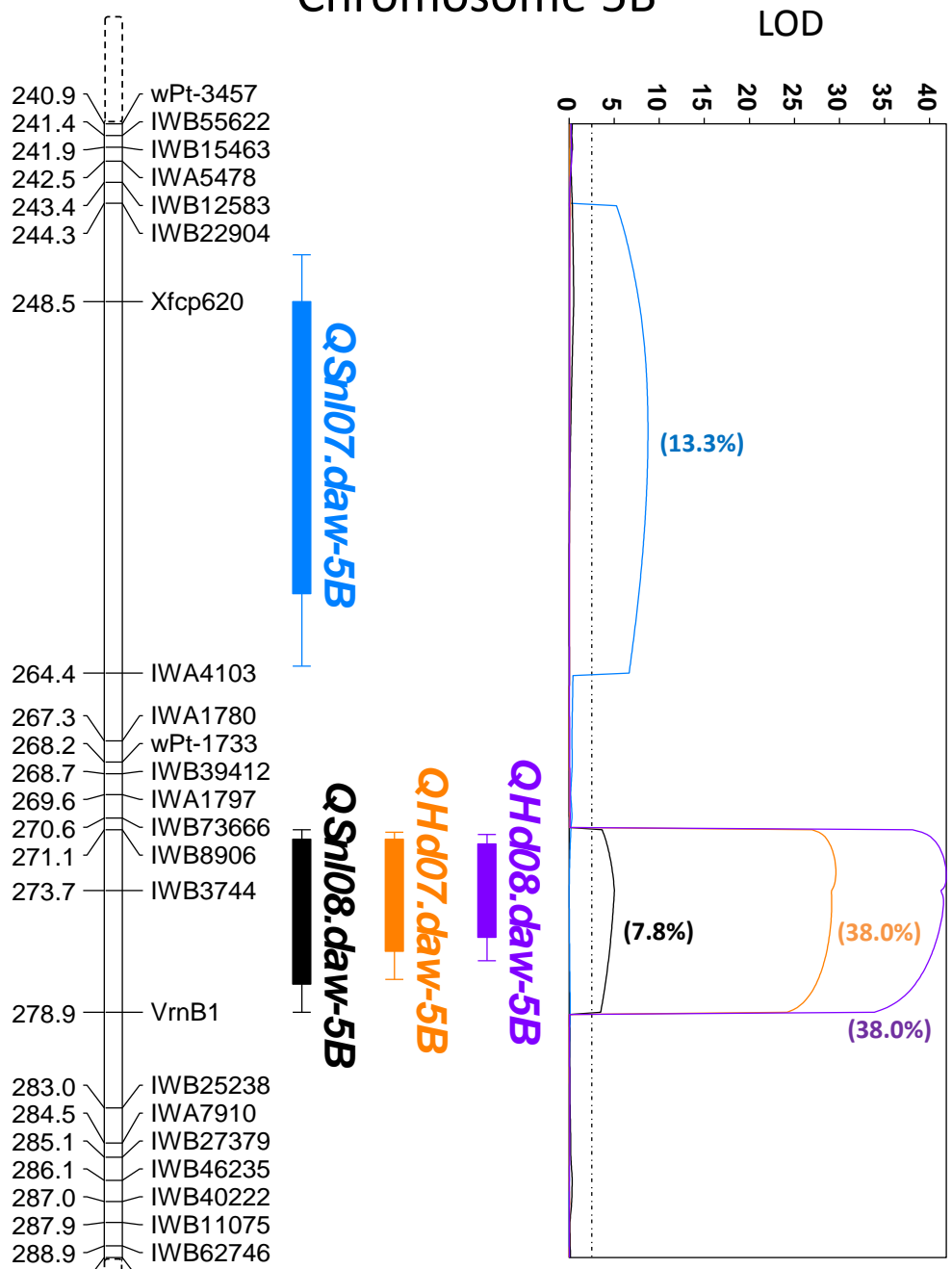

289.4

IWB56998

Chromosome 2D

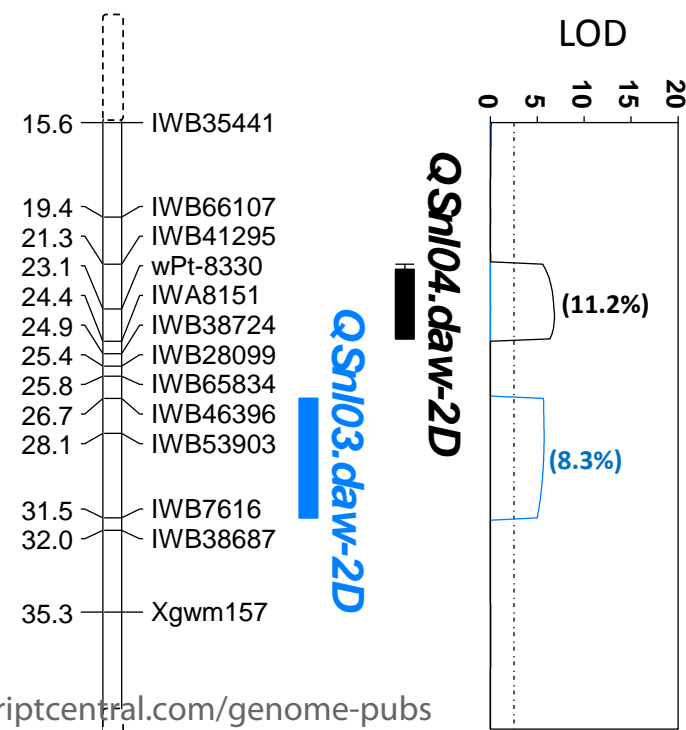




\section{Chromosome 2D}

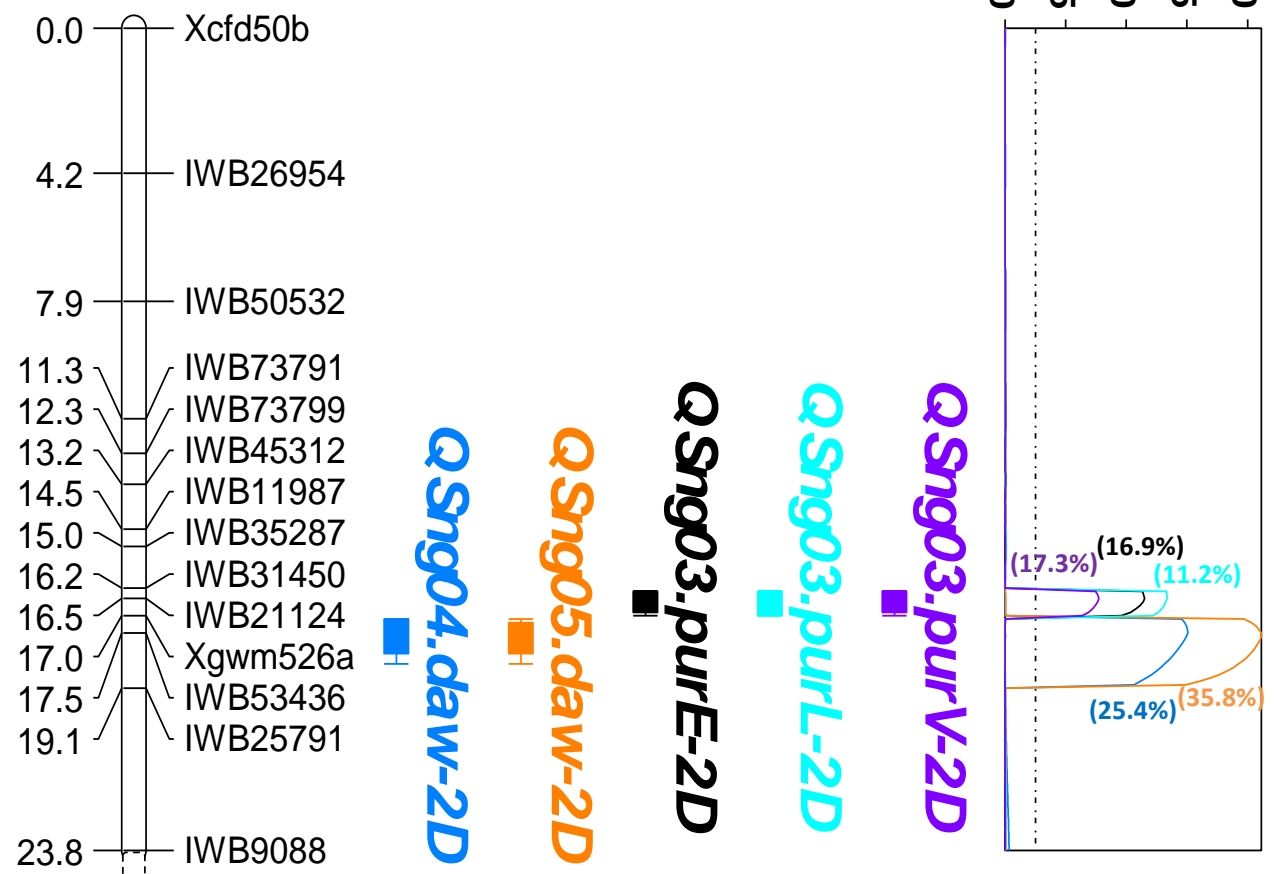

\section{Chromosome 4B}

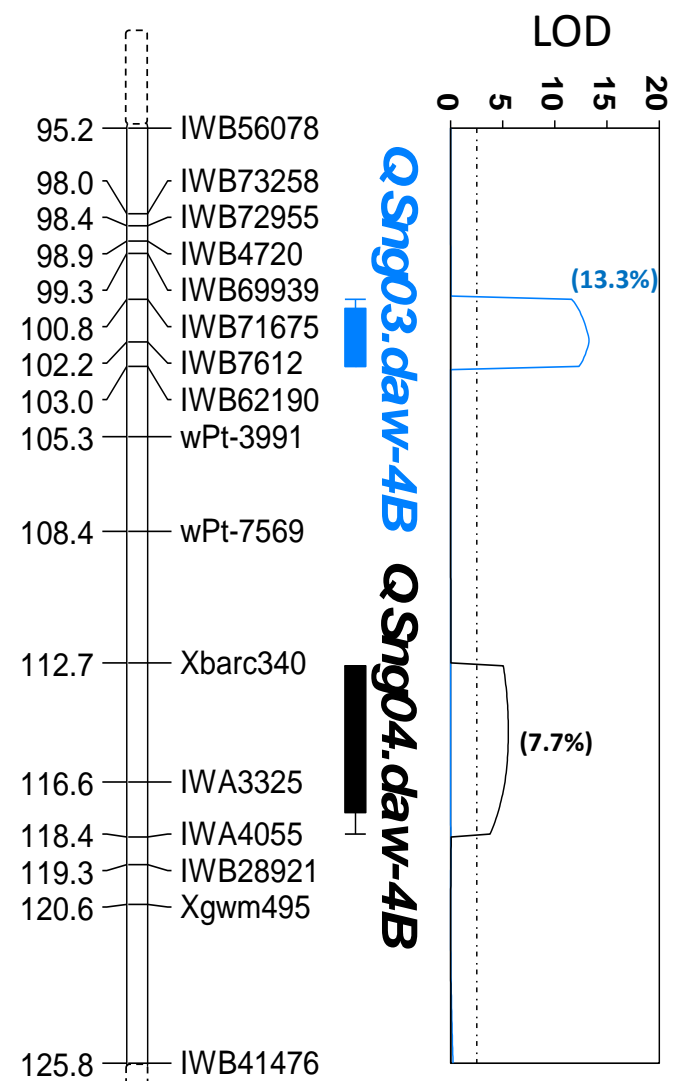

